\title{
Review
}

\section{Tumour-released exosomes and their implications in cancer immunity}

\author{
M lero ${ }^{1}$, R Valenti ${ }^{1}$, V Huber ${ }^{1}$, P Filipazzi ${ }^{1}$, G Parmiani ${ }^{2}$, S Fais ${ }^{3}$ and L Rivoltini ${ }^{\star, 1}$
}

Tumour cells release vesicular structures, defined as microvesicles or exosomes, carrying a large array of proteins from their originating cell. The expression of antigenic molecules recognized by T cells has originally suggested a role for these organelles as a cell-free antigen source for anticancer vaccines. However, recent evidence shows that tumour exosomes may also exert a broad array of detrimental effects on the immune system, ranging from apoptosis in activated antitumour T cells to impairment of monocyte differentiation into dendritic cells and induction of myeloid suppressive cells. Immunosuppressive exosomes of tumour origin can be found in neoplastic lesions and sera from cancer patients, implying a potential role of this pathway in in vivo tumour progression. Through the expression of molecules involved in angiogenesis promotion, stromal remodelling, delivery of signalling pathways through growth factor/receptor transfer, chemoresistance and genetic intercellular exchange, tumour exosomes could represent a versatile tool for moulding host environment. Hence, their secretion by neoplastic cells may in the future become a novel pathway to target for therapeutic intervention in cancer patients.

Cell Death and Differentiation (2008) 15, 80-88; doi:10.1038/sj.cdd.4402237; published online 12 October 2007

From a therapeutic point of view, cancer has been faced so far as a disease stemming from alterations of the cellular genome and affecting expression and/or function of oncogenes and tumour suppressor genes. However, it is nowadays widely accepted that tumour microenvironment plays a pivotal role in cancer development and progression. ${ }^{1,2}$ Indeed, tumour cells begin to mould the host environment, starting at early phases of the neoplastic process, to favour their survival and expansion. While some host components, such as the immune system, may initially attempt to restrict disease progression, defences are progressively blunted by the activation of suppressive pathways or even turned into tumour-promoting players. ${ }^{3}$ An almost never-ending list of phenotypical and functional defects involving different components of the immune system, together with the mechanisms by which tumour cells mediate these defects, has been published over the last two decades. Alterations ranging from dysfunctional dendritic cells (DCs), anergy or apoptosis in antitumour effector $T$ cells to the expansion of regulatory immune cell populations, including regulatory $T$ cells and myeloid suppressor cells (MSCs), induced by a broad array of soluble factors or cell-to-cell contact-mediated interactions, have been described to occur in cancer patients, although the actual contribution of these pathways in favouring in vivo progression still needs to be fully clarified in the human setting. Obviously, the complexity of the network established between tumour cells and their environment makes the identification of potential intervention aimed at disrupting these detrimental connections a difficult task.

In the following paragraphs, we want to discuss mechanisms employed by tumour cells to hide from immune recognition and promote their in vivo survival and progression. Particular attention will be given to the ability of tumour cells to secrete small vesicles (known as exosomes or microvesicles) gathering a still growing number of immunosuppressive and modulating functions. The possibility of interfering by pharmacological intervention with their formation and/or release process, and the theoretical benefits of this strategy for cancer patients, are also addressed.

\section{Tumour Cells as Mediators of Immune Dysfunctions}

Despite the self origin of most tumour antigens, the occurrence of spontaneous cancer immunity in cancer patients is a wellaccepted concept. ${ }^{4}$ This process, known as cancer immunosurveillance, represents the attempt of the host immune system to contain cancer growth in the early phases of development. ${ }^{5}$ However, this condition of equilibrium usually fails with disease progression, through escape mechanisms adopted by tumour cells to silence their immunogenic profile and survive by activating immunosuppressive/deviating pathways. ${ }^{6}$ Although the fine alterations occurring in cancer cells

${ }^{1}$ Unit of Immunotherapy of Human Tumours, Fondazione IRCCS Istituto Nazionale Tumori, Milan, Italy; ${ }^{2}$ Unit of Immunobiotherapy of Solid Tumours, San Raffaele Scientific Institute, Milan, Italy and ${ }^{3}$ Department of Drug Research and Evaluation, Pharmacogenetic, Drug Resistance, and Experimental Therapeutic Section, Istituto Superiore di Sanità, Rome, Italy

${ }^{*}$ Corresponding author: L Rivoltini, Unit of Immunotherapy of Human Tumours, Fondazione IRCCS Istituto Nazionale Tumori, Via Venezian 1, Milan 20133, Italy. Tel: 0 + 3922390 3245; Fax: + 3922390 2154; E-mail: licia.rivoltini@ istitutotumori.mi.it

Keywords: tumour exosome; immune modulation; chemotherapy; PPI

Abbreviations: APC, antigen-presenting cell; DC, dendritic cell; DEX, DC exosome; ESCRT, endosomal sorting complexes required for transport; HSP, heat-shock protein; LN, lymph node; MMP, matrix metalloproteinase; MSC, myeloid suppressor cell; MVB, multivesicular body; PPI, proton pump inhibitor

Received 18.7.07; revised 07.9.07; accepted 07.9.07; Edited by G Kroemer; published online 12.10.07 
that are responsible for the switch from immune recognition to immune suppression are only partially delineated, ${ }^{7}$ transformed cells are undeniably a sort of active reservoir of harmful molecules profoundly interfering with host functions. ${ }^{8}$ The ultimate effects of these pathways can indeed be seen at a tumour site, in draining lymph nodes (LNs) and in the peripheral circulation of cancer patients as defects virtually affecting all components of the immune system. ${ }^{9}$

Because of their key role in priming specific immune responses, DCs are supposed to represent the front line of immune defences that needs to be inactivated to avoid immunity. In this view, several studies support the existence in cancer patients of a myeloid compartment that, under tumour and microenvironment influences, shifts from effective antigen presenting cells (APCs) to negative modulators of immune responses. ${ }^{10} \mathrm{~A}$ vast array of alterations affecting the number, phenotype and function have been described in peripheral blood, draining LNs and tumour sites of cancer patients. ${ }^{11,12}$ In particular, DCs are often described as displaying altered expression of HLA-DR and co-stimulatory molecules as well as defective cytokine release, resulting in the cross-priming of tolerogenic or anergized $\mathrm{T}$ cells instead of efficient antitumour effectors. ${ }^{11-14}$ Furthermore, DCs are directly affected in their specialized ability to process and present exogenous antigens through their antigen processing machinery, thereby losing their crucial ability to prime specific $\mathrm{T}$ cells through cross-presentation. ${ }^{15}$ In addition to the lack of functional APCs, cancer patients are affected by the accumulation in peripheral blood and tumour sites ${ }^{16,17}$ of myeloid precursors arrested at an immature differentiation stage, exerting active suppression on the development of specific T cell responses, and known as MSCs. ${ }^{18}$ This series of functional aberrations affecting the myeloid compartment is deemed to be the result of multiple tumour-driven mechanisms requiring either direct interaction with tumour cells or the release of soluble factors that reach myeloid precursors in bone marrow and other tissues. ${ }^{19}$ This evidence suggests that myeloid dysfunctions may be driven in cancer patients by a systemic mechanism most likely consisting of soluble or circulating factors.

A series of molecules and pathways have been indicated as potentially responsible for these alterations, including secretion of soluble factors (such as VEGF, IL-10 and PGE2) and overexpression of molecules on tumour cell surface (for instance gangliosides). ${ }^{20-23}$ However, it is worth mentioning that soluble factors are likely to act mostly locally or in the immediate vicinity of the releasing site, and they could hardly be deemed to be the only cause of the systemic and multiple effects than can be observed in the peripheral blood or bone marrow of cancer patients. In this view, more efficient pathways for the delivery of immune suppressive/deviating signals by tumour cells to the immune system should exist to explain the systemic dysfunctions observed in cancer patients.

\section{Tumour Exosomes: a Versatile Tool of Immune Modulation}

Cancer cells are believed to mould microenvironment components and affect immune system function mainly by pathways involving cell-to-cell contact and the release of suppressive soluble factors. However, an alternative novel mechanism that is now emerging involves the active release by tumour cells of immune suppressive membrane microvesicles, also known as exosomes. ${ }^{24}$

Microvesicles or exosomes are endosome-derived organelles of 50-100 nm size, which are actively secreted through an exocytosis pathway that is used under normal as well as pathological conditions for receptor discharge and intercellular cross-talk ${ }^{24-26}$ (Figure 1). Even though exosomes have recently been found to be released by virtually all cell types, most of the studies are actually focused on exosomes secreted by reticulocytes, immune cells and tumour cells. ${ }^{24}$ Proteomic analysis of microvesicles underlined that although several molecules are shared between microvesicles of different cell origin, exosome functionality seems to be determined by specific protein content. In fact, exosomes derived from DCs (also called DEX) can elicit T cell activation in mouse model when previously loaded with tumour peptides, thanks to a set of co-stimulatory and cell adhesion molecules as well as functional MHC class II and class I molecules. ${ }^{27}$ Further in vitro observations revealed that the stimulatory effects of DEX are linked to the transfer of relevant molecules among DCs, thus amplifying stimulatory signals and inducing enhanced immune responses. ${ }^{28,29}$ In this view, DEX have recently been used in clinical trials as a potential antitumour vaccine. $^{30,31}$

Microvesicle release is exacerbated in tumour cells, as demonstrated by in vitro studies and the abundance of vesicular organelles that can be purified from plasma, ascites and pleural effusions of cancer patients. ${ }^{29,32,33}$ The initial identification of exosome release by tumour cells was envisaged as the discovery of a new cell-free source of tumour antigens for in vivo immune priming or tumour vaccine design. Indeed, exosomes are close replicas of the originating cells in terms of selected protein content and express a large array of tumour antigens when secreted by neoplastic cells. To name a few examples, melanoma-derived exosomes contain the highly immunogenic antigens MelanA/Mart-1 and gp100, those released by colon carcinoma cells express CEA and HER2, ${ }^{29,34}$ which can be detected in microvesicles secreted by breast carcinoma. This antigenic content is not only a feature of in vitro-released exosomes, but also can be found in microvesicles isolated from plasma of cancer patients as well, evidence that demonstrates the tumour origin of these organelles. Moreover, proteomic analysis has definitively proved the enrichment in exosomes of different cell origins of several members of the heat-shock protein family (HSP) (including an HSP70 and HSP90), ${ }^{35,36}$ which are known to favour APC activation through the delivery of danger signals. $^{37}$

These findings thereby inspired vaccination studies conducted in animal models, which pointed at tumour-derived exosomes as an antigenic source for T-cell priming, ${ }^{38}$ while ex vivo experiments in cancer patients were performed to demonstrate the ability of exosomes purified from ascitic fluids, to generate antitumour $T$ cells when cross-presented by autologous DCs. ${ }^{29}$

However, as tumour-released microvesicles retain a large part of the protein repertoire of the producing cells, including 


\begin{tabular}{|c|}
\hline 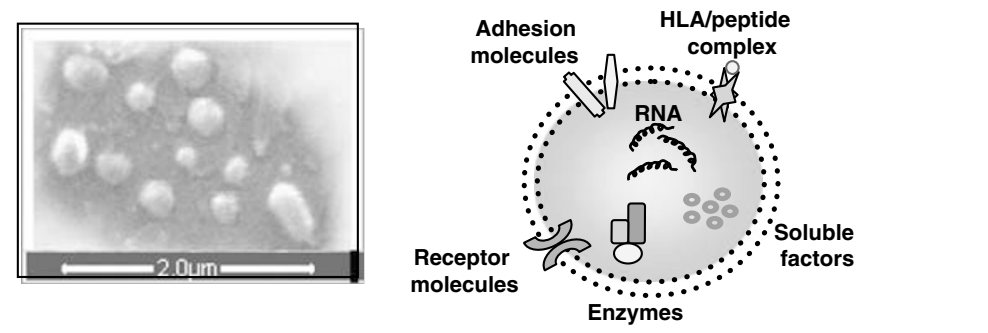 \\
\hline $\begin{array}{l}\text { Microvesicles of endosomal origin } \\
50-100 \mathrm{~nm} \text { in size }\end{array}$ \\
\hline $\begin{array}{l}\text { - Exosome-transported molecules } \\
\text { - Membrane adhesion: integrins, annexins }{ }^{[35]} \\
\text { - Membrane transport: Rab protein family }{ }^{[35]} \\
\text { - Cytoskeletal components: actin, ERM proteins }{ }^{[35]} \\
\text { - Lysosomal markers: CD63, CD81, CD82, LAMP-1/2 [53] } \\
\text { - Ag-presentation: HLA class I and II/peptide complexes }{ }^{[53]} \\
\text { - Tumour antigens: MelanA/Mart-1, gp100, CEA, HER2 }{ }^{[29,32]} \\
\text { - Death receptors: FasL, TRAIL }{ }^{[32,34]} \\
\text { - Cytokines and cognate receptors: Tfr2, TNF-a, TNFR1, TGF- }{ }^{[25,26,46]} \\
\text { - Enzymes: pyruvate kinase, enolase... }{ }^{[36]} \\
\text { - Heat Shock Proteins }{ }^{[35]} \\
\text { - mRNAs and microRNAs }{ }^{[57]} \\
\text { - Drug transporters: ATP7A, ATP7B, MRP2 }{ }^{[58]}\end{array}$ \\
\hline $\begin{array}{l}\text { Exosome secretion reported in the following human tumour } \\
\text { histotypes: } \\
\text { - Melanoma, colon carcinoma, ovarian carcinoma, breast carcinoma, head } \\
\text { and neck carcinoma, prostate carcinoma, B cell lymphoma, plasmacytoma, } \\
\text { mesothelioma } \\
\text { - Evidence of in vivo secretion (in biological fluids) in patients with } \\
\text { melanoma, colon carcinoma, prostate carcinoma, head and neck carcinoma, } \\
\text { NSCLC, renal carcinoma }\end{array}$ \\
\hline
\end{tabular}

Figure 1 Phenotypic features of exosomes secreted by human tumour cells. Tumour released exosomes or microvesicles have been characterized by several groups for their morphological features and protein content. A list of human tumour histologies in which exosomes have been isolated and analysed (in vitro or ex vivo, from biological fluids of cancer patients) is provided. Numbers indicate the concerned references

molecules involved in immune suppression and deviation, the additional effects of these organelles on antitumour immune responses would not be a surprising finding. Indeed, we found that crucial components of the immune response, such as APCs, are profoundly affected by the encounter with tumour exosomes. As a matter of fact, these microvesicles not only impair the capacity of circulating $\mathrm{CD}_{14}{ }^{+}$monocytes to differentiate into functional DCs, but they also skew the differentiation of these cells towards altered $\mathrm{CD} 14^{+}$monocytes expressing low or absent levels of HLA-DR. ${ }^{33}$ These cells, which are present in relatively high numbers in PBMCs of melanoma patients, exert suppressive activity on lymphocyte proliferation and impair the expression of effector molecules (such as perforin and IFN- $\gamma$ ) in a TGF- $\beta$-mediated fashion. $\mathrm{CD}_{14}{ }^{+} \mathrm{HLA}-\mathrm{DR}{ }^{\text {neg/low }}$ cells behave as MSC, undergoing in vivo expansion upon administration of GMCSF, a growth factor with known activating properties on myeloid-derived suppressive cells in different murine tumour models. ${ }^{39,40}$ Interestingly, the high frequency of these cells in peripheral blood is associated with a reduced induction of $\mathrm{CD}^{+} \mathrm{T}$ cell-mediated immune response after anti-melanoma vaccine administration. ${ }^{16}$ The fact that the hallmark alterations induced by tumour microvesicles on host cells in vitro can also be found in immune cells isolated from cancer patients supports the hypothesis that these negative loops can actually be established in vivo as well. ${ }^{41,42}$

As a further proof of the pleiotropic effect of tumour-secreted exosomes, it should be mentioned that tumour exosomes can also interfere directly with $T$ cell effector functions. Indeed, we have recently shown that the 
expression of bioactive FasL and TRAIL enable exosomes derived from human tumours (such as melanoma and colorectal carcinoma) to induce apoptosis in activated tumour-specific $T$ cells. This phenomenon highly resembles the one utilized under physiological conditions not only by T cells to downsize immune responses, ${ }^{43}$ but also by placenta cells that recently have been shown to promote a state of immune privilege by inducing FasL-mediated apoptosis and defects in the expression of crucial TCR signalling components (such as CD3-zeta and JAK3) in local T cells, thereby contributing to successful pregnancy. ${ }^{44,45}$ The induction of apoptosis and TCR alterations in effector T cells, which have been reported by other groups in other tumour histologies (e.g. in head and neck cancer or ovarian carcinoma), could be reproduced with microvesicles isolated from plasma of cancer patients and may help to explain the high frequency of apoptotic or CD3-zeta ${ }^{\text {neg }}$ lymphocytes that are often found in the peripheral circulation of these patients. ${ }^{46,47}$ Other immune effectors, such as for instance natural killer cells, are not spared from these negative influences as they lose their cytolytic potential, through the downmodulation of perforin expression, upon encounter with tumour-secreted microvesicles. $^{48}$

These data, together with those collected by other groups, ${ }^{41,49}$ suggest that exosomes might also act as a vehicle for suppressive signals and have negative effects on antitumour immune responses. This hypothesis appears to be in contrast with the initial evidence of immunogenicity described for tumour exosomes cross-presented by DCs. ${ }^{29,38}$ However, the final outcome of immune responses raised in the presence of tumour exosomes could strictly depend on the differentiation/maturation state of the targeted APCs. ${ }^{50}$ Hence, only in vivo studies focused on evaluating how exosome release by cancer cells affects immune functions could actually clarify the role these microvesicles play in tumour immunity.

According to our hypothesis, the immunosuppressive effects of tumour exosomes could potentially be exerted at least on two distinct steps of the process, that is, during crosspriming by DCs, with the impaired differentiation on monocytes into DCs, and at T cell level, with the release by MSCs of TGF- $\beta$, blocking proliferation and effector functions, and with the induction of apoptosis in activated cells (Figure 2). These pleiotropic effects prompt to hypothesize that interfering with exosome release by tumour cells may perhaps represent a novel strategy for simultaneously recovering multiple immune functions in cancer patients.

\section{Relationships between Exosomes and Host Environment}

Tumour-secreted exosomes are nowadays gaining increasing attention as an alternative tool of intercellular communication with extensive paracrine functions. ${ }^{51}$ Because of their doublelayer membrane being characterized by a peculiar lipid composition (highly enriched in sphingomyelin, cholesterol and the glycolipid GM3), ${ }^{52}$ exosomes might represent a more efficient tool of signal delivery as compared to soluble molecules. They should in fact (i) provide more stable conformational conditions of the protein content; (ii) increase bioactivity of the proteins expressed in their context (thanks to the maintenance of the transmembrane form of the secreted proteins); (iii) improve molecule biodistribution (for the ability of microvesicles to recirculate in body fluids and reach distant organs) and (iv) promote a more efficient interaction with target

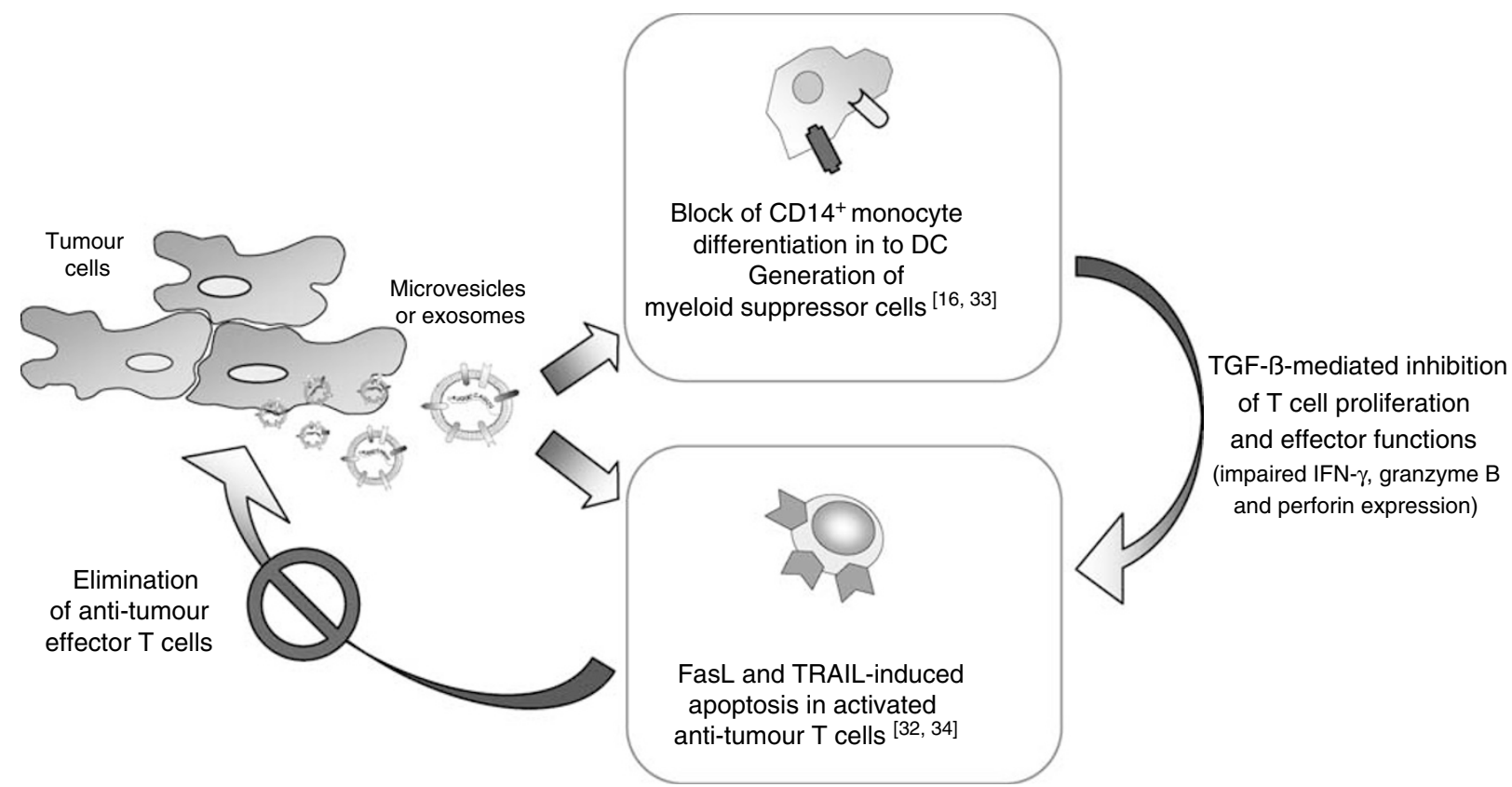

Figure 2 Negative effects of tumour-secreted exosomes on antigen-presenting cells (APCs) and T cells. Exosomes released by several human tumours (including melanoma, colon carcinoma, head and neck cancer and ovarian carcinoma) have been shown to interfere with antitumour immunity by (1) blocking monocyte differentiation into DCs and promoting the generation of myeloid suppressor cells with TGF- $\beta$-mediated suppressive activity on T-cell proliferation and function and (2) by inducing FasL-and TRAIL-mediated apoptosis in activated tumour-specific T cells. Both these pathways lead to tumour immune escape. Numbers indicate the concerned references 
cells (due to the highly fusogenic properties of exosomes). ${ }^{49}$ Altogether, these features make tumour-released exosomes an efficient platform for the in vivo transfer of cross-talk signals. As a matter of fact, the large array of bioactive molecules incorporated into tumour-produced exosomes ${ }^{24,35,36}$ prompts speculation that these organelles might potentially play a paracrine role in shaping host environment. A scheme of the hypothetical pathways involving tumour exosomes is depicted in Figure 3. Although direct evidences are still rather limited, especially in the in vivo setting, exosomes have the potential to actually transfer specific proteins to homologous and heterologous target cells for the delivery of signalling pathways. ${ }^{53-55}$ Recent studies are clearly evidencing that tumour microvesicles increase matrix metalloproteinase (MMP) secretion and

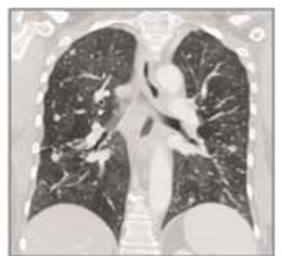

Role in autocrine signals to distant tumour cells

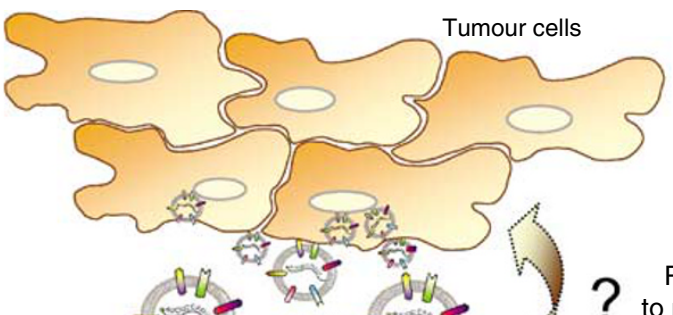

2. Role in autocrine signals neighbouring tumour cells (TNF- $\alpha /$ TNF receptors; mRNA, microRNA) ${ }^{[25,57]}$

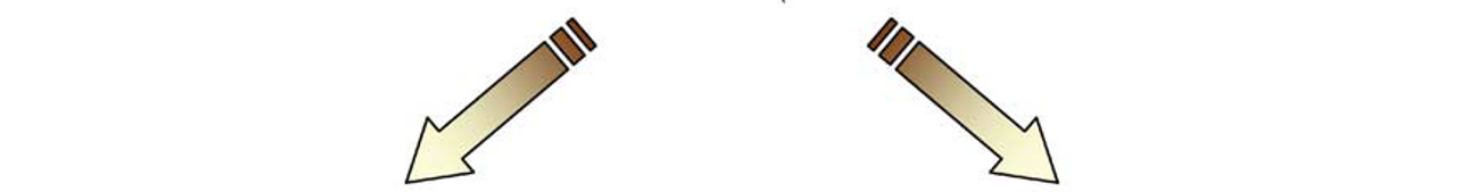

Microvesicles

or exosomes

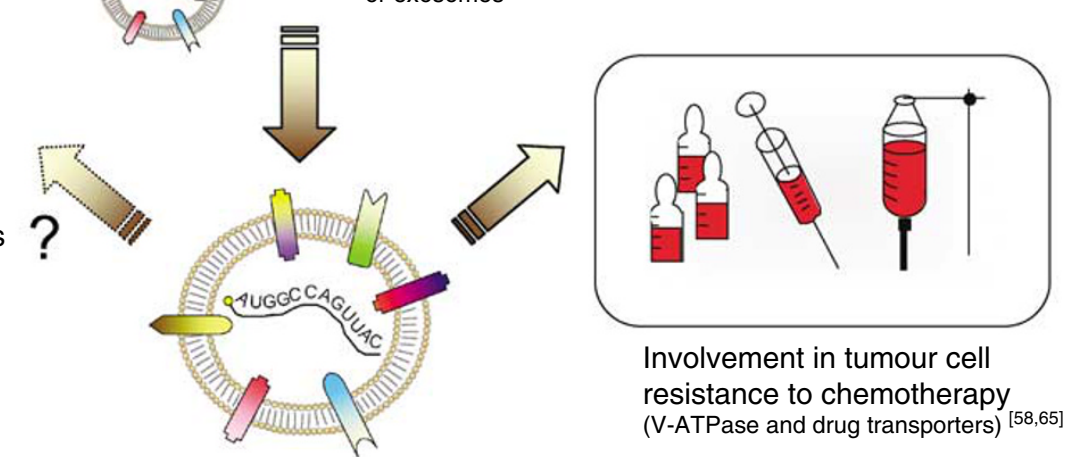

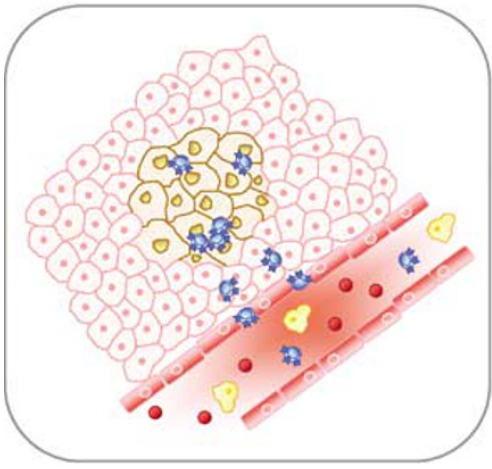

Effects on different components of the anti-tumour immune response

(FasL, TNF-a, TGF- $\beta$ ) $[25,26,34]$

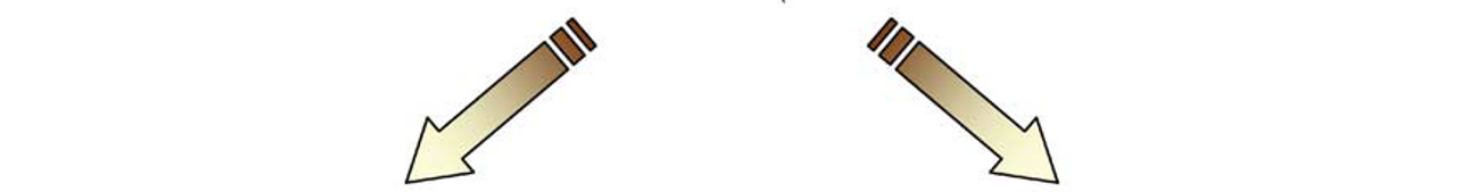


VEGF expression in target cells through the expression of proangiogenic molecules, such as members of the Tetraspanin family, thus promoting neo-angiogenesis even at tumour distant sites. ${ }^{56}$ Owing to the multifunctional protein repertoire transported by tumour exosomes, it could be hypothesized that they may also contribute to the process of stromal remodelling, thus widening the role of these microvesicles in tumour progression and metastatization process.

Similar to what was observed with exosomes of specific normal cells (e.g. immune cells and red cells), ${ }^{53}$ exosomes of tumour origin could perhaps be involved in the transfer of bioactive receptors, such as for instance HER2, ${ }^{29}$ among tumour cells or to environment cell components. This hypothesis, which has just started to be addressed in literature, is already supported by some initial results showing that tumour exosomes can indeed promote the epigenetic transfer of metastatic activity in murine models. ${ }^{55}$ In addition, preliminary data from our group are suggesting that specific growth factors and cognate receptors are transported by microvesicles released in vitro and in vivo by human melanoma cells ( $R$ Valenti et al., unpublished data). An efficient shaping of protein synthesis in target cells could also be achieved by the exosome-mediated transfer of functional mRNAs and microRNAs, recently reported to occur in transformed mastocytes. ${ }^{57}$

\section{Pharmacological Modulation of Exosome Secretion by Tumour Cells}

The identification of the role of microvesicles in shaping tumour microenvironment at different levels could offer a novel and alternative view to conceive cancer treatments. Indeed, if the immunosuppressive role of tumour exosomes should be confirmed by in vivo studies, the search for drugs interfering with exosome secretion by tumour cells might theoretically represent a potential strategy to help restore tumour immunity and impair tumour progression. However, definitive information on the mechanisms regulating microvesicle release by cancer cells are presently lacking.

Interesting insights into this process have recently been provided by studies linking tumour chemoresistance and exosome secretion. Indeed, on the basis of their biochemical features and electrostatic charges some drugs tend to accumulate into vesicular compartments and are then eliminated by vesicle shedding. ${ }^{58-60}$ This pathway has been described in melanoma, where the histotype-specific pattern of melanosome release is exploited for cisplatin elimination, with a consequent drug-mediated enhancement of vesicle shedding. ${ }^{59}$ However, cells lacking melanosomes, such as ovarian carcinoma cells, display the same features and get rid of cytotoxic drugs, such as doxorubicin, through exosome discharge ${ }^{61}$ thanks to alterations in the endosomal compartment and lysosomal structures. ${ }^{59}$ On the basis of these data, treatment of chemoresistant tumour cells with cisplatin or doxorubicin could result in exacerbated exosome shedding, ${ }^{59,61}$ perhaps through the p53 pathway that was recently associated with exosome release. ${ }^{62}$ In contrast, opposite effects could be expected with drugs interfering with microtubule stability, such as taxanes and vinca alkaloids. Indeed, preliminary data from our laboratory, investigating the involvement of cytoskeleton components in endosomal stability and/or direct exocytosis pathway, are showing that treatment of melanoma cells with non-cytotoxic doses of microtubule-targeting drugs (paclitaxel and vinblastine) could reduce microvesicle release ( $\mathrm{M}$ lero, unpublished observations). Similarly, alterations of the lysosomal compartment are observed in breast cancer cells treated with additional microtubule-disturbing drugs, such as vincristine, ${ }^{63}$ in which increased total lysosomal volume most likely results from a defective lysosome exocytosis.

Another promising tool may be represented by drugs interfering with the activity of enzymatic efflux pumps expressed on acidic vacuoles, called vacuolar-ATPases, and associated to exosome pathway in $C$. elegans model (V-ATPases). ${ }^{64}$ These molecules, which have been primarily studied for their implications in tumour chemoresistance, are indeed expressed at exosomal level ${ }^{64}$ and could be involved in the altered trafficking and function of the endolysosomal compartment. Indeed, we have shown that inhibition of this pathway through proton pump inhibitors (PPIs) interferes with traffic of acidic vesicles, resulting in their sequestration within the cytoplasm, and improving chemosensitivity in melanoma cells. ${ }^{65}$ This suggests that anti-acidic treatment may interfere with exosome release and consequently be a potential factor limiting the harmful effects that these microvesicles exert on the immune system. Interestingly, treatment with PPIs leads to apoptotic cell death of tumour cells when utilized at high dose as single agent. ${ }^{66}$

Benefits from modulation of exosome secretion could also come from qualitatively modulating the protein composition of secreted microvesicles with drugs altering the biological features of tumour vesicles. A potential pathway involved in exosome biogenesis has recently been suggested as being linked to a protein complex, known as ESCRT (endosomal sorting complexes required for transport) machinery. This complex is responsible for sorting proteins into the intralumenal vesicles of multivesicular bodies (MVBs). Through a ubiquitination-dependent mechanism, proteins assigned to lysosome degradation are tagged to MVBs to be degraded after fusion with lysosomes. ${ }^{67}$ Modification of ubiquitinated proteins might help modulate exosome protein composition and thus biological activity, as recently reported using curcumin, a natural polyphenol that has been shown to reduce exosome immunosuppressive functions when administered to breast carcinoma cells. ${ }^{68}$ A simplified summary of the drug interfering with exosome release by tumour cells is illustrated in Figure 4.

Independently, from the pharmacological tool utilized for diminishing exosome release by tumour cells, the crucial point is then to prove that interfering with microvesicle secretion results in improved immunogenicity of cancer cells. Studies are presently in progress to address this hypothesis in animal tumour models or in cancer patients treated with PPIs. Preliminary data from our laboratory actually show that PPIs treatment of melanoma cells, which significantly impair exosome release, is associated with a significant increase of the cytolytic and the cytokine release potential of melanoma-specific $\mathrm{CD}^{+} \mathrm{T}$ cells (M lero, unpublished observation). 


\begin{tabular}{|c|c|c|c|c|}
\hline $\begin{array}{c}\text { Drug } \\
\downarrow \\
\text { Possible } \\
\text { mechanism }\end{array}$ & $\begin{array}{c}\text { Cisplatin, doxorubicin }[58,59,61] \\
\text { p53 activation }[62]\end{array}$ & $\begin{array}{c}\text { Taxol, vinca alkaloids* } \\
\downarrow \\
\text { Endo-lysosomal stability }\end{array}$ & $\begin{array}{l}\text { Curcumin [68] } \\
\qquad \\
\text { Protein sorting }\end{array}$ & $\begin{array}{c}\mathrm{PPI}^{\star} \\
\downarrow \\
\text { V-ATPase }\end{array}$ \\
\hline $\begin{array}{l}\text { Intra- } \\
\text { cellular } \\
\text { target }\end{array}$ & & 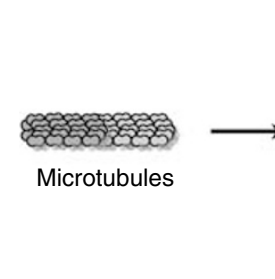 & Multivesicular $\begin{array}{c}\text { bodies } \\
\text { bo }\end{array}$ & $\begin{array}{l}\mathrm{O}_{\mathrm{O}} \mathrm{O}_{\mathrm{O}} \\
\text { Secreted } \\
\text { exosomes }\end{array}$ \\
\hline $\begin{array}{l}\text { Potential } \\
\text { effects on } \\
\text { exosomes }\end{array}$ & $\begin{array}{c}\text { Increased } \\
\text { exosome } \\
\text { release }\end{array}$ & $\begin{array}{c}\text { Decreased } \\
\text { exosome } \\
\text { release }\end{array}$ & $\begin{array}{l}\text { Modulation } \\
\text { exosome } \\
\text { composition }\end{array}$ & $\begin{array}{c}\text { Decreased } \\
\text { exosome } \\
\text { release }\end{array}$ \\
\hline
\end{tabular}

Figure 4 Drugs potentially interfering with exosome release by tumour cells. Several chemotherapeutic drugs may interfere with microvesicle release by tumour cells at different levels of the process of exosome formation and with different outcomes. Treatment with non-cytotoxic doses of cisplatin and doxorubicin, possibly through p53 activation, enhances exosome release, while drugs interfering with microtubule activity (such as taxanes and vinca alkaloids) reduce their secretion. Molecules acting on multivesicular body (MVB) assembly can change exosome protein composition. Proton pump inhibitors (PPIs) decrease microvesicle release through an unknown mechanism, possibly related to direct activity on lysosome stability or to their influence on tumour $\mathrm{pH}$. ( ${ }^{*} \mathrm{M}$ lero et al., unpublished observation). Numbers indicate the concerned references

\section{Concluding Remarks}

On the basis of current information presently available, the release of cellular material by transformed cells, either as a consequence of cell necrosis/apoptosis or as an effect of exosome active secretion, could no longer be considered as a bona fide antigenic source for effective $T$ cell cross-priming. Despite a high percentage of antitumour vaccines presently under clinical evaluation (like those based on gene-modified allogeneic tumour cells, tumour-derived lysate or apoptotic bodies) relying on the 'natural' immunogenicity of cancer cells as a source of antigenic material, ${ }^{69-72}$ recent studies have clearly reported that dying tumour cells need to display specific features, such as exposure of calreticulin on surface debris, to be phagocytosed by APCs and thus gain immunogenicity. ${ }^{73}$ But aside from these defined conditions, the abundance of molecules with immune suppressive/deviating properties which might be associated with tumour antigenic material, could profoundly influence the final outcome of the immune response, especially in the complex setting of cancer patients, and it could partially explain why the clinical efficacy of vaccine approaches has so far been rather unsatisfactory.

The use of combination treatments associating immunotherapy with more conventional strategies, such as chemotherapy, has recently been seen as a 'must' for achieving better response rates. However, inducing tumour cell apoptosis by cytotoxic drugs does not necessarily guarantee that the released antigenic material would mediate effective APC activation and T cell cross-priming ${ }^{73}$ In addition to improving cancer immunity by promoting immunogenic death, one alternative strategy could be to create, by pharmacological intervention, a more favourable environment for $T$ cell activation, that is by getting rid of those negative factors that are known to restrain antitumour immunity. Despite the negative impact of tumour exosomes on the immune system still needing to be definitively demonstrated in vivo, microvesicle secretion could indeed represent a potential pathway to target for achieving this goal. Indeed, by reducing the bioavailability of tumour microvesicles through pharmacological intervention, it could be speculated that multiple functions of cancer immunity could be simultaneously recovered: DC differentiation from monocytes could be unblocked, the influence of MSCs could be limited and anti-proliferative/ proapoptotic signals for $\mathrm{T}$ cells could be reduced. In contrast, other approaches targeting one single regulatory mechanism, such as those aimed at eliminating regulatory $T$ cells (by toxins targeting $\mathrm{CD}_{2} 5^{+}$cells, low-dose cyclophosphamide, nonmyeloablative chemotherapy or anti-CTLA4 antibody) ${ }^{74-76}$, might have more limited effects, especially in the absence of definitive information about the actual role that the different regulatory pathways play in cancer patients. On the contrary, low doses of selected chemotherapeutics, or the use of unexpected drugs such as PPIs, may help interrupt the detrimental effects mediated by tumour-released exosomes on host environment. This could become a more pleiotropic and promising strategy for achieving long-lasting control of tumour growth in cancer patients in the future.

Acknowledgements. We are grateful to Drs. Fabio Castiglioni and Erica Dugnani (Unit of Molecular Biology, Fondazione IRCCS Istituto Nazionale Tumori, Milan, Italy) for the excellent graphical support, and to Dr. Antonello Villa (MIA Consortium, University of Bicocca, Milan, Italy) for scanning electron microscopy images. This work was supported by grants from the European Community Cancer Immunology and Immunotherapy contract no. 518234), the Italian Ministry of Industry, University and Research (Rome, Italy: grant FIRB RBNE017B4C), the Italian Association for Cancer Research. R Valenti was supported by a scholarship from the Foundation for Cancer Research (Milan, Italy).

1. Balkwill $F$, Charles KA, Mantovani A. Smoldering and polarized inflammation in the initiation and promotion of malignant disease. Cancer Cell 2005; 7: 211-217.

2. Lin WW, Karin M. A cytokine-mediated link between innate immunity, inflammation, and cancer. J Clin Invest 2007; 117: 1175-11831. 
3. Rivoltini L, Canese $\mathrm{P}$, Huber V, lero M, Pilla L, Valenti R et al. Escape strategies and reasons for failure in the interaction between tumour cells and the immune system: how can we tilt the balance towards immune-mediated cancer control? Expert Opin Biol The 2005; 5: 463-476.

4. Houghton AN. Cancer antigens: immune recognition of self and altered self. $J$ Exp Med 1994; 180: 1-4

5. Dunn GP, Old LJ, Schreiber RD. The immunobiology of cancer immunosurveillance and immunoediting. Immunity 2004; 21: 137-148.

6. Zou W. Immunosuppressive networks in the tumour environment and their therapeutic relevance. Nat Rev Cancer 2005; 5: 263-274.

7. Hahn WC, Weinberg RA. Rules for making human tumor cells. N Engl J Med 2002; 347: 1593-1603.

8. Gajewski TF, Meng Y, Blank C, Brown I, Kacha A, Kline J et al. Immune resistance orchestrated by the tumor microenvironment. Immunol Rev 2006; 213: 131-145.

9. Rabinovich GA, Gabrilovich D, Sotomayor EM. Immunosuppressive strategies that are mediated by tumor cells. Annu Rev Immunol 2007; 25: 267-296.

10. Gabrilovich D. Mechanisms and functional significance of tumour-induced dendritic-cell defects. Nat Rev Immunol 2004; 4: 941-952.

11. Do TH, Johnsen HE, Kjaersgaard E, Taaning E, Svane IM. Impaired circulating myeloid DCs from myeloma patients. Cytotherapy 2004; 6: 196-203.

12. Vakkila J, Thomson AW, Vettenranta K, Sariola H, Saarinen-Pinkala UM. Dendritic cell subsets in childhood and in children with cancer: relation to age and disease prognosis. Clin Exp Immunol 2004; 135: 455-461.

13. Della Bella S, Gennaro M, Vaccari M, Ferraris C, Nicola S, Riva A et al. Altered maturation of peripheral blood dendritic cells in patients with breast cancer. Br J Cancer 2003; 89: 1463-1472.

14. Chaux $P$, Moutet $M$, Faivre J, Martin F, Martin M. Inflammatory cells infiltrating human colorectal carcinomas express HLA class II but not B7-1 and B7-2 costimulatory molecules of the T-cell activation. Lab Invest 1996; 74: 975-983.

15. Whiteside TL, Stanson J, Shurin MR, Ferrone S. Antigen-processing machinery in human dendritic cells: up-regulation by maturation and down-regulation by tumor cells. J Immunol 2004; 173: 1526-1534

16. Filipazzi P, Valenti R, Huber V, Pilla L, Canese P, lero M et al. Identification of a new subse of myeloid suppressor cells in peripheral blood of melanoma patients with modulation by granulocyte-macrophage colony-stimulation factor-based antitumor vaccine. J Clin Oncol 2007; 25: 2546-2553.

17. Bronte V, Kasic T, Gri G, Gallana K, Borsellino G, Marigo I et al. Boosting antitumo responses of $\mathrm{T}$ lymphocytes infiltrating human prostate cancers. J Exp Med 2005; 201 1257-1268

18. Serafini P, Carbley R, Noonan KA, Tan G, Bronte V, Borrello I. High-dose granulocytemacrophage colony-stimulating factor-producing vaccines impair the immune response through the recruitment of myeloid suppressor cells. Cancer Res 2004; 64: 6337-6343.

19. Almand B, Resser JR, Lindman B, Nadaf S, Clark JI, Kwon ED et al. Clinical significance of defective dendritic cell differentiation in cancer. Clin Cancer Res 2000; 6: 1755-1766.

20. Ohm JE, Carbone DP. VEGF as a mediator of tumor-associated immunodeficiency. Immunol Res 2001; 23: 263-272

21. Prguet-Navarro J, Sportouch M, Popa I, Berthier O, Schmitt D, Portoukalian J. Gangliosides from human melanoma tumors impair dendritic cell differentiation from monocytes and induce their apoptosis. J Immunol 2003; 170: 3488-3494.

22. Gosset $P$, Bureau F, Angeli V, Pichavant M, Faveeuw $C$, Tonnel AB et al. Prostaglandin D2 affects the maturation of human monocyte-derived dendritic cells: consequence on the polarization of naive Th cells. J Immunol 2003; 170: 4943-4952.

23. Brown R, Murray A, Pope B, Sze DM, Gibson J, Ho PJ et al. Either interleukin-12 or interferon-gamma can correct the dendritic cell defect induced by transforming growth factor beta in patients with myeloma. Br J Haematol 2004; 125: 743-748.

24. van Niel G, Porto-Carreiro I, Simoes S, Raposo G. Exosomes: a common pathway for a specialized function. J Biochem (Tokyo) 2006; 140: 13-21.

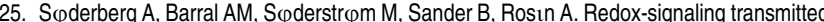
in trans to neighboring cells by melanoma-derived TNF-containing exosomes. Free Radic Biol Med 2007; 43: 90-99.

26. Clayton A, Mitchell JP, Court J, Mason MD, Tabi Z. Human tumor-derived exosomes selectively impair lymphocyte responses to interleukin-2. Cancer Res 2007; 67: 7458-7466.

27. Zitvogel L, Regnault A, Lozier A, Wolfers J, Flament C, Tenza D et al. Eradication of established murine tumors using a novel cell-free vaccine: dendritic cell-derived exosomes. Nat Med 1998; 4: 594-600

28. Thıry C, Zitvogel L, Amigorena S. Exosomes: composition, biogenesis and function. Nat Rev Immunol 2002; 2: 569-579.

29. Andre F, Schartz NE, Movassagh M, Flament $C$, Pautier P, Morice $P$ et al. Malignan effusions and immunogenic tumour-derived exosomes. Lancet 2002; 360: 295-305.

30. Escudier B, Dorval T, Chaput N, Andre F, Caby MP, Novault S et al. Vaccination of metastatic melanoma patients with autologous dendritic cell (DC) derived-exosomes: results of the first phase I clinical trial. J Transl Med 2005; 3: 10

31. Morse MA, Garst J, Osada T, Khan S, Hobeika A, Clay TM. A phase I study of dexosome immunotherapy in patients with advanced non-small cell lung cancer. J TransI Med 2005; $\mathbf{~ : ~} 9$.

32. Huber V, Fais S, lero M, Lugini L, Canese $P$, Squarcina $P$ et al. Human colorectal cance cells induce T-cell death through release of proapoptotic microvesicles: role in immune escape. Gastroenterology 2005; 128: 1796-1804.
33. Valenti R, Huber V, Filipazzi P, Pilla L, Sovena G, Villa A et al. Human tumor-released microvesicles promote the differentiation of myeloid cells with transforming growth factorbeta-mediated suppressive activity on T lymphocytes. Cancer Res 2006; 66: 9290-9298.

34. Andreola G, Rivoltini L, Castelli C, Huber V, Perego P, Deho P et al. Induction of lymphocyte apoptosis by tumor cell secretion of FasL-bearing microvesicles. J Exp Med 2002; 195: 1303-1316.

35. Mears R, Craven RA, Hanrahan S, Totty N, Upton C, Young SL et al. Proteomic analysis of melanoma-derived exosomes by two-dimensional polyacrylamide gel electrophoresis and mass spectrometry. Proteomics 2004; 4: 4019-4031.

36. Hegmans JP, Bard MP, Hemmes A, Luider TM, Kleijmeer MJ, Prins JB et al. Proteomic analysis of exosomes secreted by human mesothelioma cells. Am J Pathol 2004; 164: 1807-1815.

37. Srivastava P. Interaction of heat shock proteins with peptides and antigen presenting cells: chaperoning of the innate and adaptive immune responses. Annu Rev Immunol 2002; 20: 395-425.

38. Wolfers J, Lozier A, Raposo G, Regnault A, Thıry C, Masurier C et al. Tumor-derived exosomes are a source of shared tumor rejection antigens for CTL cross-priming. Nat Med 2001; 7: 297-303.

39. Serafini P, Borrello I, Bronte V. Myeloid suppressor cells in cancer: recruitment, phenotype, properties, and mechanisms of immune suppression. Semin Cancer Biol 2006; 16: 53-65.

40. Parmiani G, Castelli C, Pilla L, Santinami M, Colombo MP, Rivoltini L. Opposite immune functions of GM-CSF administered as vaccine adjuvant in cancer patients. Ann Oncol 2007; 18: 226-232.

41. Valenti R, Huber V, lero M, Filipazzi P, Parmiani G, Rivoltini L. Tumor-released microvesicles as vehicles of immunosuppression. Cancer Res 2007; 67: 2912-2915.

42. Wieckowski $\mathrm{E}$, Whiteside TL. Human tumor-derived vs dendritic cell-derived exosomes have distinct biologic roles and molecular profiles. Immunol Res 2006; 16: 415-421.

43. Monle $\mathrm{n}$ I, Martvnez-Lorenzo MJ, Monteagudo L, Lasierra P, Taulıs M, Iturralde M et al. Differential secretion of Fas ligand- or APO2 ligand/TNF-related apoptosis-inducing ligandcarrying microvesicles during activation-induced death of human T cells. J Immunol 2001; 167: 6736-6744.

44. Sabapatha A, Gercel-Taylor C, Taylor DD. Specific isolation of placenta-derived exosomes from the circulation of pregnant women and their immunoregulatory consequences. $A m \mathrm{~J}$ Reprod Immunol 2006; 56: 345-355.

45. Taylor DD, Akyol S, Gercel-Taylor C. Pregnancy-associated exosomes and their modulation of T cell signaling. J Immunol 2006; 176: 1534-1542.

46. Taylor DD, Gernel-Taylor C. Tumour-derived exosomes and their role in cancerassociated T-cell signalling defects. Br J Cancer 2005; 92: 305-311.

47. Kim JW, Wieckowski E, Taylor DD, Reichert TE, Watkins S, Whiteside TL. Fas ligandpositive membranous vesicles isolated from sera of patients with oral cancer induce apoptosis of activated T lymphocytes. Clin Cancer Res 2005; 11: 1010-1020.

48. Liu C, Yu S, Zinn K, Wang J, Zhang L, Jia Y et al. Murine mammary carcinoma exosomes promote tumor growth by suppression of NK cell function. $J$ Immunol 2006; 176: $1375-1385$.

49. Whiteside TL. Tumour-derived exosomes or microvesicles: another mechanism of tumour escape from the host immune system? Br J Cancer 2005; 92: 209-211.

50. Yu S, Liu C, Su K, Wang J, Liu Y, Zhang L et al. Tumor exosomes inhibit differentiation of bone marrow dendritic cells. J Immunol 2007; 178: 6867-6875.

51. Fivrier B, Raposo G. Exosomes: endosomal-derived vesicles shipping extracellular messages. Curr Opin Cell Biol 2004; 16: 415-421.

52. Subra $C$, Laulagnier K, Perret $B$, Record $M$. Exosome lipidomics unravels lipid sorting at the level of multivesicular bodies. Biochimie 2007; 89: 205-212.

53. Denzer K, Kleijmeer MJ, Heijnen HF, Stoorvogel W, Geuze HJ. Exosome: from internal vesicle of the multivesicular body to intercellular signaling device. J Cell Sci 2000; 113: 3365-3374.

54. Calzolari A, Raggi C, Deaglio S, Sposi NM, Stafsnes M, Fecchi K et al. TfR2 localizes in lipid raft domains and is released in exosomes to activate signal transduction along the MAPK pathway. J Cell Sci 2006; 119: 4486-4498.

55. Hao S, Ye Z, Li F, Meng Q, Qureshi M, Yang J et al. Epigenetic transfer of metastatic activity by uptake of highly metastatic B16 melanoma cell-released exosomes. Exp Oncol 2006; 28: 126-131.

56. Gesierich S, Berezovskiy I, Ryschich E, Z $\varphi$ ller M. Systemic induction of the angiogenesis switch by the tetraspanin D61A/CO-029. Cancer Res 2006; 66: 7083-7094.

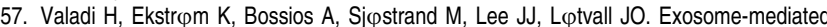
transfer of mRNAs and microRNAs is a novel mechanism of genetic exchange between cells. Nat Cell Biol 2007; 9: 654-659.

58. Safaei R, Larson BJ, Cheng TC, Gibson MA, Otani S, Naerdemann W et al. Abnormal lysosomal trafficking and enhanced exosomal export of cisplatin in drug-resistant human ovarian carcinoma cells. Mol Cancer Ther 2005; 4: 1595-1604.

59. Chen KG, Valencia JC, Lai B, Zhang G, Paterson JK, Rouzaud F et al. Melanosomal sequestration of cytotoxic drugs contributes to the intractability of malignant melanomas. Proc Natl Acad Sci USA 2006; 103: 9903-9907.

60. Kroemer G, Jaattela M. Lysosomes and autophagy in cell death control. Nat Rev Cancer 2005; 5: 886-897.

61. Shedden K, Xie XT, Chandaroy P, Chang YT, Rosania GR. Expulsion of small molecules in vesicles shed by cancer cells: association with gene expression and chemosensitivity profiles. Cancer Res 2003: 63: 4331-4337. 
62. Yu X, Harris SL, Levine AJ. The regulation of exosome secretion: a novel function of the p53 protein. Cancer Res 2006; 66: 4795-4801.

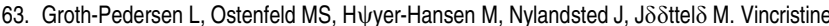
induces dramatic lysosomal changes and sensitizes cancer cells to lysosome-destabilizing siramesine. Cancer Res 2007; 67: 2217-2225.

64. Liıgeois S, Benedetto A, Garnier JM, Schwab Y, Labouesse M. The V0-ATPase mediates apical secretion of exosomes containing Hedgehog-related proteins in Caenorhabditis elegans. J Cell Biol 2006; 173: 949-961.

65. Luciani F, Spada M, De Milito A, Molinari A, Rivoltini L, Montinaro A et al. Effect of proton pump inhibitor pretreatment on resistance of solid tumors to cytotoxic drugs. J Natl Cancer Inst 2004; 96: 1702-1713.

66. De Milito A, lessi E, Logozzi MA, Lozupone F, Spada M, Marino ML et al. Proton pump inhibitors induce apoptosis of human B cell tumors through a caspaseindependent mechanism involving reactive oxygen species. Cancer Res 2007; 67 : 5408-5417.

67. de Gassart A, Giminard C, Hoekstra D, Vidal M. Exosome secretion: the art of reutilizing nonrecycled proteins? Traffic 2004; 5: 896-903

68. Zhang HG, Kim H, Liu C, Yu S, Wang J, Grizzle WE et al. Curcumin reverses breast tumor exosomes mediated immune suppression of NK cell tumor cytotoxicity. Biochim Biophys Acta 2007; 1773: 1116-11123.

69. Soiffer R, Lynch T, Mihm M, Jung K, Rhuda C, Schmollinger JC et al. Vaccination with irradiated autologous melanoma cells engineered to secrete human granulocyte- macrophage colony-stimulating factor generates potent antitumor immunity in patients with metastatic melanoma. Proc Natl Acad Sci USA 1998; 95: 13141-13146.

70. Palucka AK, Ueno $\mathrm{H}$, Connolly J, Kerneis-Norvell $\mathrm{F}$, Blanck JP, Johnston DA et al. Dendritic cells loaded with killed allogeneic melanoma cells can induce objective clinical responses and MART-1 specific CD8+ T-cell immunity. J Immunother (1997) 2006; 29 : 545-557.

71. Maio M, Fonsatti E, Lamaj E, Altomonte M, Cattarossi I, Santantonio C et al. Vaccination of stage IV patients with allogeneic IL-4- or IL-2-gene-transduced melanoma cells generates functional antibodies against vaccinating and autologous melanoma cells. Cancer Immunol Immunother 2002; 51: 9-14.

72. Heiser A, Coleman D, Dannull J, Yancey D, Maurice MA, Lallas CD et al. Autologous dendritic cells transfected with prostate-specific antigen RNA stimulate CTL responses against metastatic prostate tumors. J Clin Invest 2002; 109: 409-417.

73. Obeid M, Tesniere A, Ghiringhelli F, Fimia GM, Apetoh L, Perfettini $\mathrm{JL}$ et al. Calreticulin exposure dictates the immunogenicity of cancer cell death. Nat Med 2007; 13: 54-61.

74. Muranski P, Boni A, Wrzesinski C, Citrin DE, Rosenberg SA, Childs R et al. Increased intensity lymphodepletion and adoptive immunotherapy - how far can we go? Nat Clin Pract Oncol 2006; 3: 668-681.

75. Peggs KS, Quezada SA, Korman AJ, Allison JP. Principles and use of anti-CTLA4 antibody in human cancer immunotherapy. Curr Opin Immunol 2006; 18: 206-213.

76. Frankel AE, Fleming DR, Powell BL, Gartenhaus R. DAB389IL2 (ONTAK) fusion protein therapy of chronic lymphocytic leukaemia. Exp Opin Biol Ther 2003; 3: 179-186. 